\title{
SERP1 is a novel marker of poor prognosis in pancreatic ductal adenocarcinoma patients via anti-apoptosis and regulating SRPRB/NF- $\mathrm{KB}$ axis
}

\author{
QIANG MA ${ }^{1,3^{*}}$, XIUXIU WU ${ }^{2 *}$, JING WU $^{4}$, ZHIYONG LIANG ${ }^{1,3}$ and TONGHUA LIU ${ }^{1,3}$ \\ Departments of ${ }^{1}$ Pathology and ${ }^{2}$ Respiratory Medicine, Peking Union Medical College Hospital; \\ ${ }^{3}$ Molecular Pathology Research Centre, Chinese Academy of Medical Sciences and Peking Union Medical College, \\ Beijing 100730; ${ }^{4}$ Department of Medical Imaging, Beijing Huairou Hospital, \\ University of Chinese Academy of Science, Beijing 101400, P.R. China
}

Received June 3, 2017; Accepted July 31, 2017

DOI: 10.3892/ijo.2017.4111

\begin{abstract}
Stress associated endoplasmic reticulum protein 1 (SERP1), can cause accumulation of unfolded proteins in ER stress. However, studies on the role of SERP1 in pancreatic ductal adenocarcinoma (PDAC) are still incomplete. The present study aimed at identifying whether SERP1 acts as a potential novel prognostic marker of PDAC, and analyzed its possible mechanism. GEO database analysis showed SERP1 was significantly upregulated in PDAC tissues, and strongly associated with advanced clinical stage of PDAC patients from TCGA database. Univariate and multivariate Cox regression analysis further revealed SERP1 high expression was an independent factor for the prognosis of PDAC. Gene set enrichment analysis (GSEA) revealed that SERP1 was mainly involved in regulating cell apoptosis and nuclear factor- $\kappa \mathrm{B}(\mathrm{NF}-\kappa \mathrm{B})$ signaling pathway, and downregulated SERP1 significantly promoted PANC-1 cell apoptosis. To further explore its possible mechanism, protein-protein interaction (PPI) and gene ontology (GO) analysis showed the functions of proteins interacting with SERP1 were mainly enriched in regulating cell apoptosis, and SRP receptor $\beta$ subunit (SRPRB) was the core of the whole PPI network. The expression of SERP1
\end{abstract}

Correspondence to: Prof. Zhiyong Liang or Prof. Tonghua Liu, Department of Pathology, Peking Union Medical College Hospital, Chinese Academy of Medical Sciences and Peking Union Medical College, 1 Shuifuyuan, Wangfujing Street, Beijing 100730, P.R. China

E-mail: liangzhiyong1220@yahoo.com

E-mail: liutonghua_pumc@163.com

*Contributed equally

Abbreviations: SERP1, stress associated endoplasmic reticulum protein 1; SRPRB, SRP receptor $\beta$ subunit; PDAC, pancreatic ductal adenocarcinoma; ER, endoplasmic reticulum

Key words: stress associated endoplasmic reticulum protein 1, pancreatic cancer, prognostic marker, SRPRB, apoptosis was negatively correlated with SRPRB expression. In vitro, downregulated SERP1 significantly increased SRPRB expression. Furthermore, upregulated SRPRB could increase cell apoptosis rate and decreased the expression level of $\mathrm{NF}-\kappa \mathrm{B}$ and the phosphorylation NF- $\mathrm{NB}$. The above results indicated that SERP1 as a potential novel prognostic marker of PDAC probably via regulating cell apoptosis and NF- $\kappa \mathrm{B}$ activation, which may be associated with SRPRB.

\section{Introduction}

Pancreatic cancer is one of the most lethal human cancers, and it is usually discovered and diagnosed at its advanced stage (1). Pancreatic ductal adenocarcinoma (PDAC) is the most common subtype of pancreatic cancer, with a low 5-year survival rate of $7.7 \%$ (2). Despite considerable advances in neoadjuvant chemotherapy, surgical techniques and perioperative care, the prognosis for PDAC has not improved significantly (3). Hence, prognosis markers are very important for PDAC patients, they provide valuable prognostic and treatment information.

Recently, a growing number of prognostic markers have been discovered, including mRNAs, non-coding RNA, circulating tumor DNA and tumor-derived exosomes (3-7). These prognostic biomarkers associate with tumor clinical stage, overall survival (OS) time, and disease-free survival (DFS) time by regulating tumor biological behavior. For example, integrin $\beta 1$ modulates tumor resistance to gemcitabine and serves as an independent prognostic factor in PDAC (8). Regenerating family member proteins promote acinar-toductal metaplasia and act as novel diagnostic and prognostic markers in PDAC (9). Vav guanine nucleotide exchange factor 3 and DIX domain containing 1 are linked to poor prognosis of pancreatic cancers and promote the proliferation, motility and invasiveness of pancreatic cancer cells $(10,11)$. However, there are no prognostic markers for use in the clinic. Thus, there is still a growing need for prognostic markers and therapeutic targets to improve the outcomes of PDAC patients.

In the present study, we found that stress associated endoplasmic reticulum protein 1 (SERP1) was 
significantly upregulated in PDAC tissues. SERP1, also known as ribosome-associated membrane protein 4 (RAMP4), is a Sec61-associated polypeptide that is induced by ER stress (12). High level of SERP1 was correlated with advanced stage and poor prognosis of PDAC. SERP1 downregulation promoted cell apoptosis via upregulating apoptosis-related protein SRP receptor $\beta$ subunit (SRPRB) expression and inhibiting nuclear factor $-\kappa \mathrm{B}(\mathrm{NF}-\kappa \mathrm{B})$ activation. Previous studies have verified that $\mathrm{NF}-\kappa \mathrm{B}$ is a known regulator of anti-apoptotic molecules $(13,14)$, is constitutively activated by phosphorylation modification in various tumors including PDAC (15-17). $\mathrm{NF}-\kappa \mathrm{B}$ phosphorylation exist in PDAC cells such as PANC-1, BxPC-3 and AsPC-1, and contributing to their resistance to apoptosis and high metastatic potential (14). The inhibition of $\mathrm{NF}-\kappa \mathrm{B}$ phosphorylation has been shown to sensitize cells to apoptosis in pancreatic cancer cells (14). Our results are the first to show SERP1 is involved in regulating NF- $\kappa \mathrm{B}$ activation and apoptosis, helping to elucidate the possible mechanism of SERP1 in predicting prognosis of PDAC.

\section{Materials and methods}

Database analysis. The mRNA expression data of 14 pairs of PDAC and adjacent tissues were obtained from GEO profiles database (http://www.ncbi.nlm.nih.gov/geo/, GSE41368, GSE16515). Mutation information and clinical data were downloaded from cBioPortal database (www.cbioportal.org). The TCGA data on mRNA (RNASeq V2) levels in PDAC patients were obtained from https://cancergenome.nih.gov/. SERP1, SRPRB and NF- $\kappa B$ mRNA level were used in the present study. The IHC-based protein expression data including high-resolution images were viewed and downloaded from the Human Protein Atlas web portal (www.proteinatlas.org). The sum IOD of IHC images were measured by Image-Pro Plus software (version 6.0; Media Cybernetics).

Gene set enrichment analysis (GSEA). The association between SERP1 and biological processes/ signaling pathway gene set was analyzed using Gene set enrichment analysis (GSEA v2.2, http://www.broad.mit.edu/gsea/). GSEA calculates a gene set enrichment score (ES) that estimates genes from pre-defined gene set. Thresholds for significance were determined by permutation analysis (1,000 permutations). A gene set is considered significantly enriched when the P-value is $<0.05$.

Cell culture and siRNA transfection. Human PDAC cell line PANC-1 was purchased from the Cell Resource Center of Beijing Xiehe (Beijing, China) and cultivated in an incubator at $37^{\circ} \mathrm{C}$ with $5 \% \mathrm{CO}_{2}$. PANC-1 cells were maintained in high-glucose Dulbecco's modified Eagle's medium (DMEM; Cell Resource Center of Beijing Xiehe, Beijing, China) supplemented with $10 \%$ fetal bovine serum (FBS; HyClone Laboratories, Inc., Logan, UT, USA) as well as penicillin (100 U/ml; Thermo Fisher Scientific, San Jose, CA, USA). The small interfering RNA targeting SERP1 and its negative control (NC) sequences were designed and synthesized by GenePharma Biotech, Co., Ltd. (Shanghai, China). The expression plasmid pcDNA3.0-SRPRB and corresponding control plasmid were preserved in our laboratory. Lipofectamine 2000 (Invitrogen, Carlsbad, CA, USA) was used for siRNA and plasmid transfection according to the manufacturer's protocol. After $48 \mathrm{~h}$ of transfection, cells were used for subsequent experiments.

Apoptosis assay. Annexin V-FITC apoptosis detection kit I (BD Biosciences, San Jose, CA, USA) was used to detect cell apoptosis. PANC-1 cells were washed twice with cold phosphate-buffered saline, and then cells were resuspend in Annexin $\mathrm{V}$ binding buffer at a concentration of $0.5 \times 10^{7}$ cells/ $\mathrm{ml}$. Transfer of cell suspension $(100 \mu \mathrm{l})$ was made to a $1 \mathrm{ml}$ test tube, and $5 \mu \mathrm{l}$ FITC Annexin V and $10 \mu \mathrm{l}$ propidium iodide solution was added to the test tube. The cells were gently vortexed and incubated for $15 \mathrm{~min}$ at room temperature $\left(25^{\circ} \mathrm{C}\right)$ in the dark. Then $400 \mu \mathrm{l}$ of Annexin $\mathrm{V}$ binding buffer was added to each tube, and analyzed by flow cytometry (Accuri C6; BD Biosciences, Franklin Lakes, NJ, USA) within 1 h.

Western blot analysis. Cells were collected and lysed in radioimmunoprecipitation buffer (Beijing CoWin Biotech Co., Ltd., Beijing, China) with protease inhibitors for $30 \mathrm{~min}$ to extract total proteins from cells with SRPRB overexpression or SERP1 knockdown. Protein levels were quantified by bicinchoninic acid assays (Beijing CoWin Biotech Co., Ltd.). Thirty micrograms of protein from each sample was resolved by $12 \%$ sodium dodecyl sulphate-polyacrylamide gel electrophoresis (Beijing CoWin Biotech Co., Ltd.). Proteins were transferred to nitrocellulose membranes (Sigma-Aldrich, St. Louis, MO, USA), which were blocked for $1 \mathrm{~h}$ in bovine serum albumin blocking buffer (Invitrogen). Subsequently, the membranes were incubated overnight at $4^{\circ} \mathrm{C}$ with primary antibodies targeting SERP1 (1:1,000 dilution, cat. no. ab130974; Abcam, Cambridge, MA, USA), SRPRB (1:300 dilution, cat. no. D223153; Sangon Biotech), phospho-NF- $\kappa$ B p65 (Ser536)(1:1,000 dilution, cat. no. 3033, Cell Signaling Technology, Beverly, MA, USA), NF-кB p65 (1:1,000 dilution, cat. no. 8242, Cell Signaling Technology), or GAPDH (1:1,000 dilution, cat. no. 70699; Abcam), were followed by incubation with an HRP-conjugated goat antirabbit secondary antibody (1:10,000 dilution, cat. no. CW0103; Beijing CoWin Biotech Co., Ltd.) for $1 \mathrm{~h}$ at room temperature. Immunocomplexes were detected using an enhanced chemiluminescence kit (Thermo Fischer Scientific), and images were analyzed using ImageJ software (version 1.62; National Institute of Health, Bethesda, MD, USA).

Protein-protein interaction (PPI) network construction. Search Tool for the Retrieval of Interacting Genes/Proteins (STRING; Search Tool for the Retrieval of Interacting Genes, http://string-db.org/) is a database of known and predicted protein interactions that may aid in the comprehensive description of cellular mechanisms and functions (18). The PPI network interacting with SERP1 in pancreatic cancer was constructed using the STRING database.

Gene ontology (GO) analysis. To explore the functional annotation enrichment of genes interacted with SERP1, GO analysis to determine clusters of these mRNAs with enriched molecular functions were performed. We used the database for annotation, visualization and integrated discovery (DAVID) v6.7 online tool (http://david.abcc.ncifcrf.gov) to functionally annotate input genes, classify gene functions, and identify 

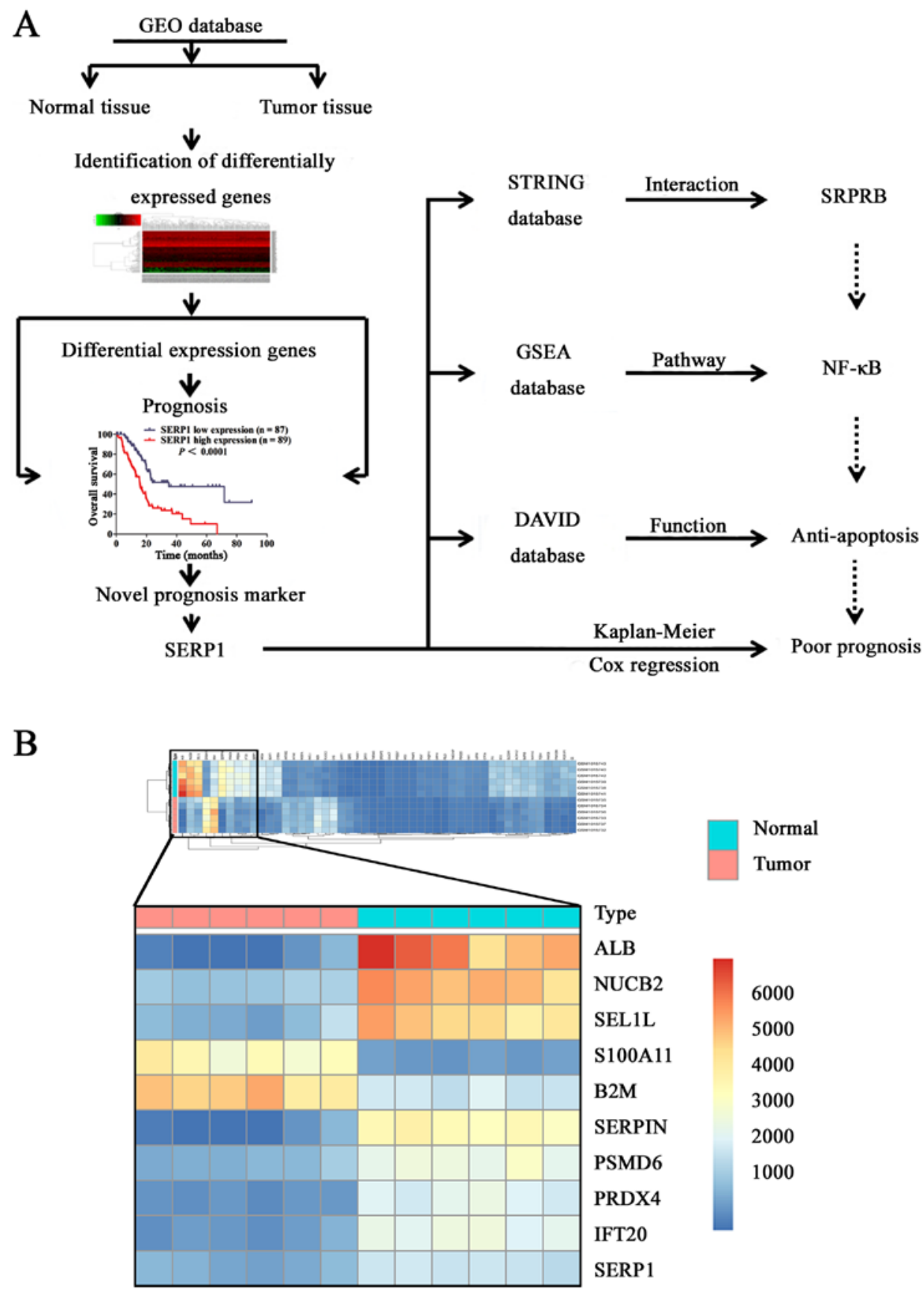

Figure 1. Flow chart of the derivation and function analysis of stress associated endoplasmic reticulum protein 1 (SERP1). (A) Flow chart. (B) Heat map of differentially expressed mRNAs in pancreatic ductal adenocarcinoma (PDAC) from GEO profiles database. The 10 mRNAs with the most significant difference are shown.

gene conversions, and to perform the GO analyses. A P-value of $<0.05$ was considered significant (19).

Statistical analysis. The statistical analysis was carried out using the SPSS 21.0 (SPSS Inc., Chicago, IL, USA) and GraphPad Prism 5 (GraphPad Software Inc., San Diego, CA, USA). mRNA expression data, IHC and apoptosis rate were analyzed using independent sample t-tests. Survival curves between different groups were obtained from Kaplan-Meier method and log-rank test. After the univariate analysis, Cox proportional hazards model was used to identify the independent prognostic factors for DFS and OS. P-values $<0.05$ were considered statistically significant.

\section{Results}

Expression level of SERPI is upregulated in PDAC tissues. As shown in flow chart of derivation and functional analysis of SERP1 (Fig. 1A), to identify the novel differentially expressed mRNAs related with PDAC occurrence and development, six pairs of PDAC tissues and adjacent normal pancreas tissues from GEO database were compared, top 10 significantly differently expressed mRNAs $(\mathrm{P}<0.01 ; \mathrm{FC}>1$ and $\mathrm{FC}<-1)$ are shown in Fig. 1B. To evaluate the correlation of differentially expressed mRNAs with prognosis, we performed survival analysis of top $10 \mathrm{mRNAs}$. Among the 10 dysregulated mRNAs, SERP1, S100A11 and SEL1L were strongly 
A

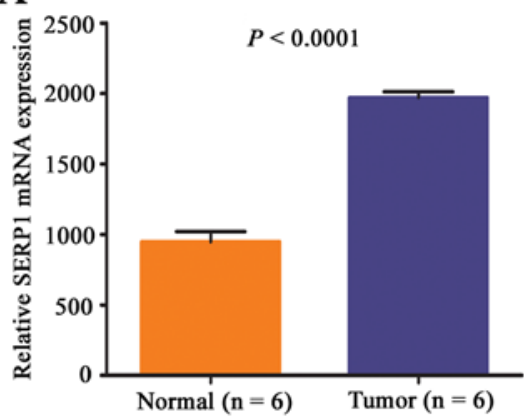

C

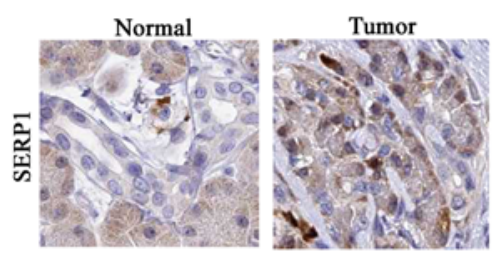

B

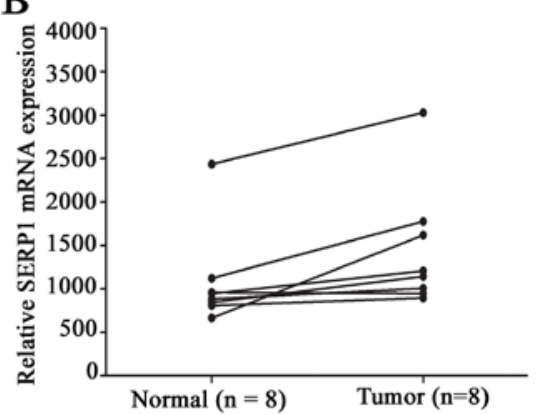

D

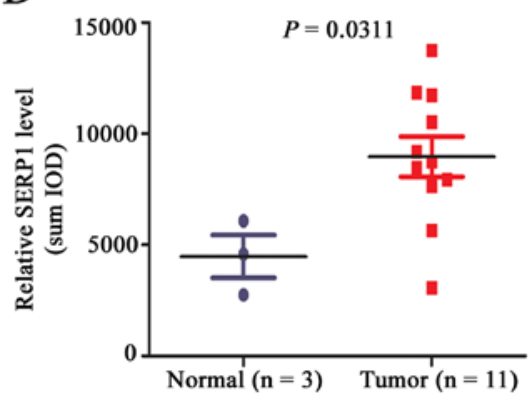

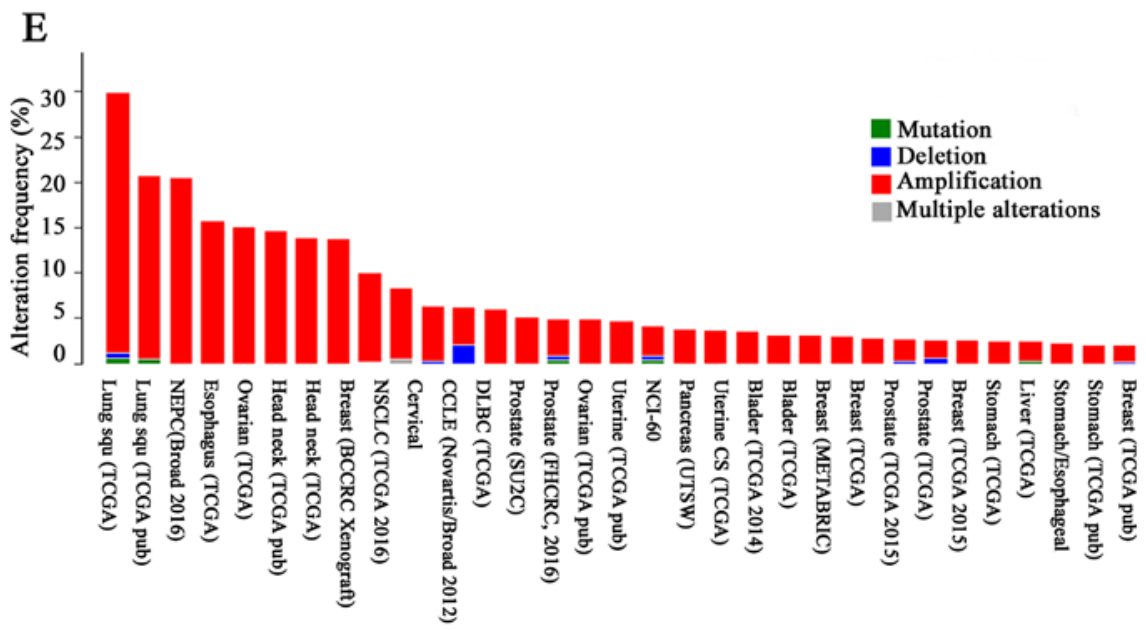

Figure 2. Expression level of the stress associated endoplasmic reticulum protein 1 (SERP1) in pancreatic ductal adenocarcinoma (PDAC) tissues is upregulated. (A) SERP1 was upregulated in six pairs of PDAC tissues compared with adjacent normal tissues. (B) The expression of SERP1 in eight pairs of PDAC tissues was upregulated compared with adjacent normal tissues from GEO database. (C and D) The Human Protein Atlas database results revealed that the expression level of SERP1 were upregulated in PDAC tissues compared with normal tissues. (E) Amplification of SERP1 was found in various tumors, including PDAC.

correlated with survival time. However, SERP1 was one of rarely reported upregulated mRNAs in PDAC (Fig. 2A), and attracted our interest. Furthermore, SERP1 was again substantially upregulated in PDAC tissues by GEO (n=8) (Fig. 2B). In Human Protein Atlas web portal database, we equally observed that SERP1 was significantly upregulated in PDAC tissues $(n=11)$ compared to normal ductal epithelial cells of pancreas $(n=3)$, and it was localized mainly in the cytoplasm of tumor cells (Fig. 2C and D). Analysis of molecular genetic alterations revealed that amplification of SERP1 exist in numerous malignant tumors, including PDAC, and we presume amplification may be a potential cause of high expression of SERP1 in PDAC (Fig. 2E).

Upregulated SERPI is associated with advanced stage and poor prognosis of PDAC. To further elucidate the role of
SERP1 in pancreatic cancer progression, the expression level of SERP1 in different stages of pancreatic cancer patients was analyzed by TCGA database. The results revealed that with the increase of $\mathrm{T}$ stage $(\mathrm{P}=0.0098)$ and clinical stage $(\mathrm{P}<0.0001)$ in PDAC, but not $\mathrm{N}$ stage $(\mathrm{P}<0.2902)$, SERP1 expression level increased accordingly (Fig. 3A-C). Next, constituent ratio analysis was performed in an expanded set of 173 primary PDAC tissues and 10 normal samples, revealing that none of the cases exhibited high expression level for SERP1 in normal pancreas tissues. However, in I/II and III/IV stage of pancreatic cancer tissues, 80/166 (56\%) of cases and 7/7 (100\%) of cases showed higher expression level for SERP1 comparing to normal tissues $(\mathrm{P}<0.01)$ (Fig. 3D). The above results suggested that SERP1 was upregulated in PDAC and associated with advanced stage, which may have prognostic significance for PDAC patients. 

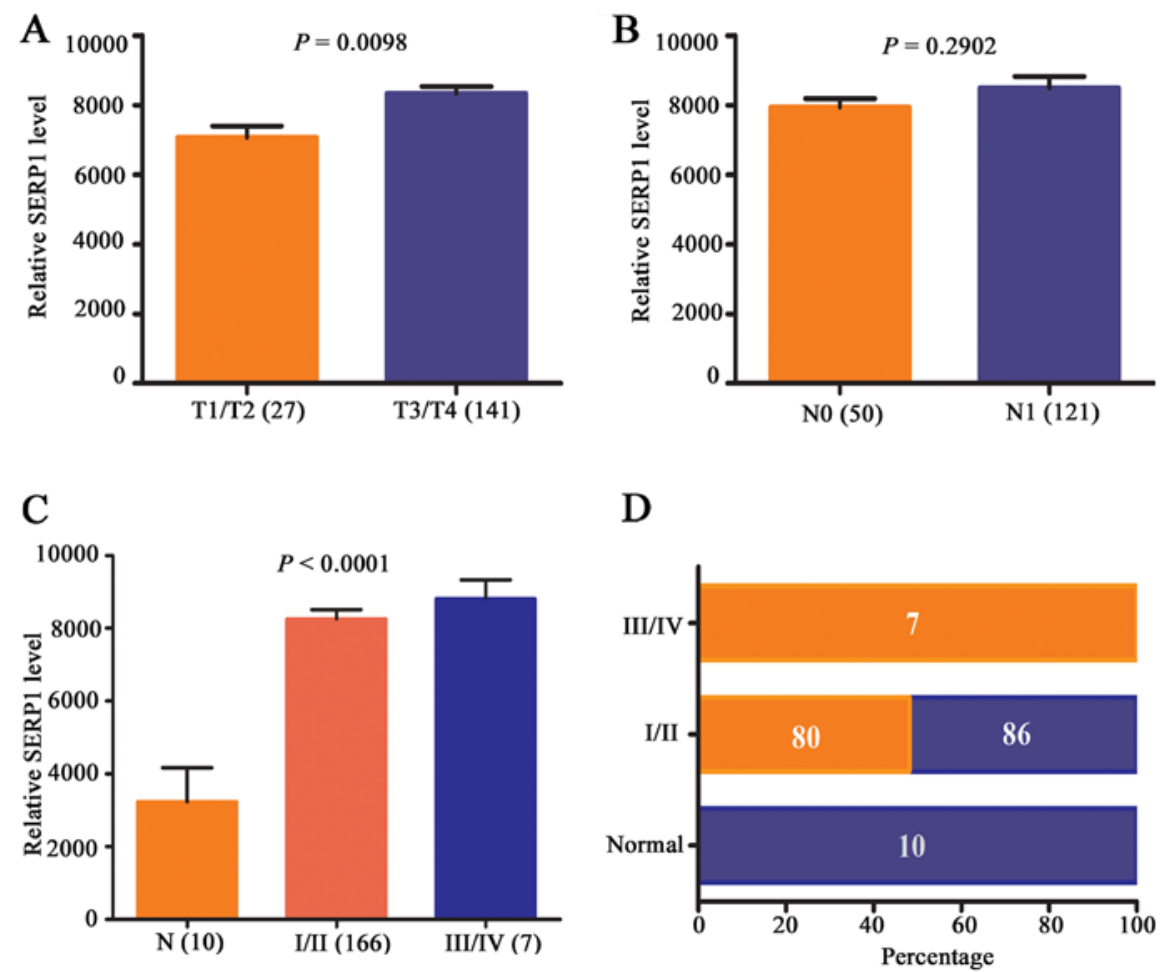

Figure 3. Correlation between expression level of the stress associated endoplasmic reticulum protein 1 (SERP1) and progression of pancreatic ductal adenocarcinoma (PDAC) patients. (A and B) The expression level of SERP1 in T3/4 stage PDAC specimen was significantly higher than in T1/2 stage, while there was no significant difference between SERP1 and $\mathrm{N}$ stage. (C) Correlation analysis was performed between expression level of SERP1 and clinical stages. SERP1 was correspondingly increased with the increase of clinical AJCC stage of PDAC. (D) Constituent ratio with high level of SERP1 was drastically different between normal samples and I/II or III/IV stage of PDAC patients.

Next, patients were divided into SERP1 high expression and SERP1 low expression groups, prognostic role of SERP1 was further investigated. Kaplan-Meier curve showed that SERP1 high expression group had shorter OS and DFS compared with SERP1 low expression group in terms of survival duration $(\mathrm{P}<0.05)$ (Fig. 4A and B), particularly in OS. Next, patients were divided into good and poor survival groups, and results revealed that the expression level of SERP1 was higher in patients with shorter DFS ( $\mathrm{P}=0.0420)$ (Fig. 4C and D). Moreover, multivariate Cox regression analysis further revealed SERP1 was an independent prognostic marker for PDAC patients (Fig. 4E and F).

Downregulated SERP1 promotes PDAC cell apoptosis. In order to gain a better understanding of the potential mechanisms of SERP1 influencing prognosis of PDAC, GSEA was performed to elucidate the functions and signaling pathways involved in these differentially expressed genes in PDAC. GSEA results showed that the gene sets related to apoptosis were enriched in PDAC tissues, including SERP1 (Fig. 5A). In vitro, si-SERP1 and corresponding negative control were transfected into PANC-1 cells. The expression level of SERP1 was significantly decreased in PANC-1 cells transfected with SERP1 siRNA (Fig. 5B), which promoted cell apoptosis compared with negative control group ( $\mathrm{P}=0.0068)$ (Fig. $5 \mathrm{C}$ and $\mathrm{D})$.

Downregulated SERP1 increases apoptosis related protein $S R P R B$ expression. Owing to protein-protein interaction playing an important role in regulating tumor biological characteristics, STRING was used to find the interaction genes with
SERP1. As Fig. 6A and B showed, these genes include SRPRB, BCL10, CLASP2, GRAMD3, GTSE1, REEP2, SCTR, VIMP, SEC61B and SERPINE. We further performed GO analysis for these interaction genes. Consistent with GSEA analysis, we noted that these genes were especially enriched in functions of regulating cell death regulation and apoptosis (Fig. 6C). Especially, apoptosis related protein SRP receptor $\beta$ subunit (SRPRB) was the highest ranked interaction genes with SERP1 (Fig. 6B), and was one of significantly co-expressed genes with SERP1 $($ Pearson $=0.63)($ Table I).

To explore the relationship between SERP1 and SRPRB, the Human Protein Atlas Database was applied. In clinical level, previous results found that SERP1 upregulated in PDAC tissues. On the contrary, SRPRB protein expression levels were significantly downregulated $(\mathrm{P}=0.0199)$ in $\mathrm{PDAC}$ tissue compared with normal pancreas tissues, it was also localized mainly in the cytoplasm of tumor cells. Interestingly, SRPRB was obviously upregulated in stromal fibroblasts of tumor tissues, but almost no expression was found of the SRPRB gene in stromal fibroblasts of normal pancreas $(\mathrm{P}=0.0090)$ (Fig. 7A and B). In vitro, si-SERP1 was transfected into PANC-1 cells and the expression level of SRPRB was increased with SERP1 downregulation in PANC-1 cells (Fig. 7C). Next, in order to identify the effect of upregulated SRPRB on PDAC cell apoptosis, pcDNA3.0-SRPRB and corresponding negative control were transfected into PANC-1 cells, respectively. The SRPRB expression level of PANC-1 cells transfected with SRPRB overexpression plasmid was upregulated (Fig. 7D), and the apoptosis rate of PANC-1 was obviously increased ( $\mathrm{P}=0.0016)$ (Fig. 7E and F). The above 

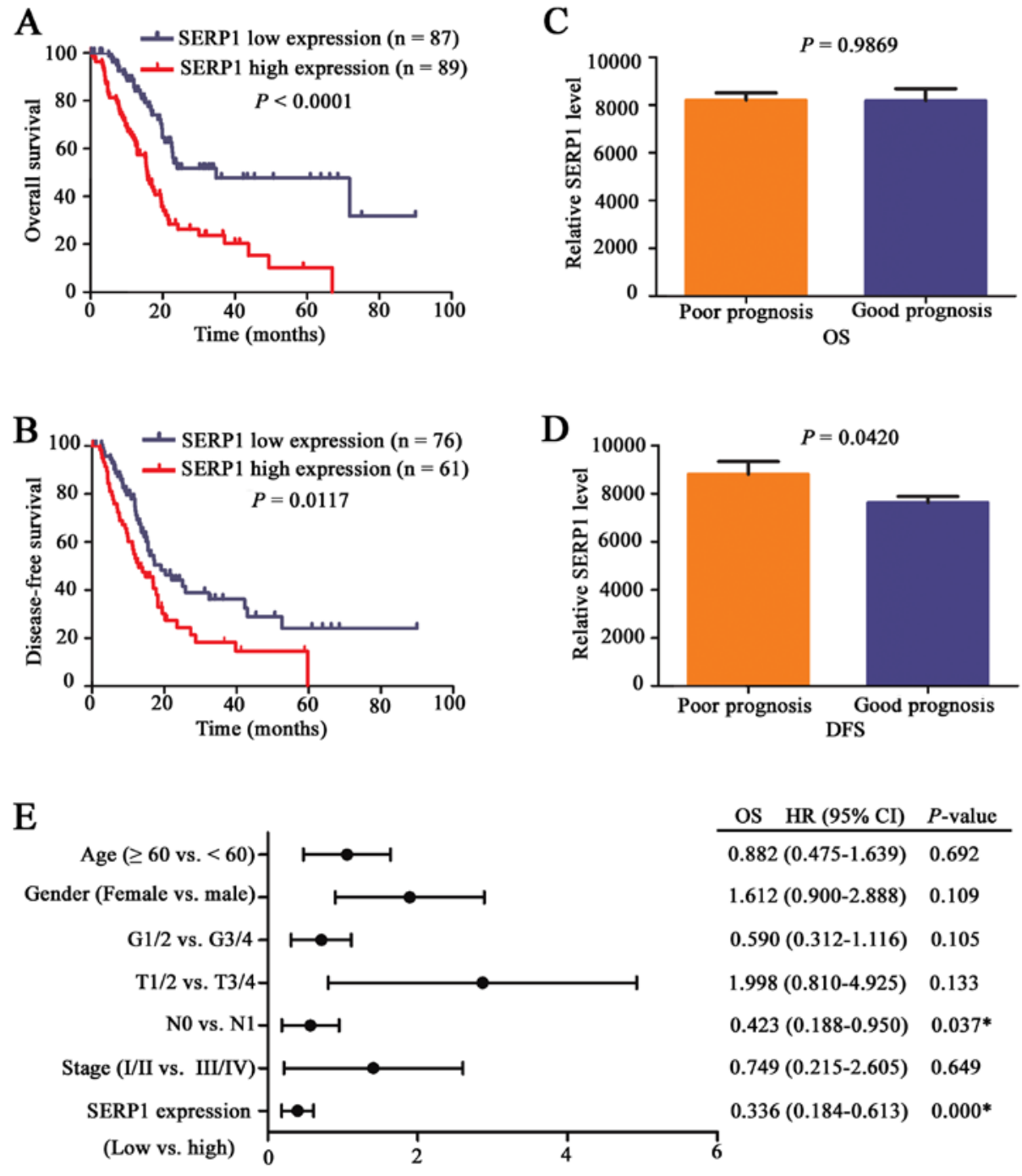

F

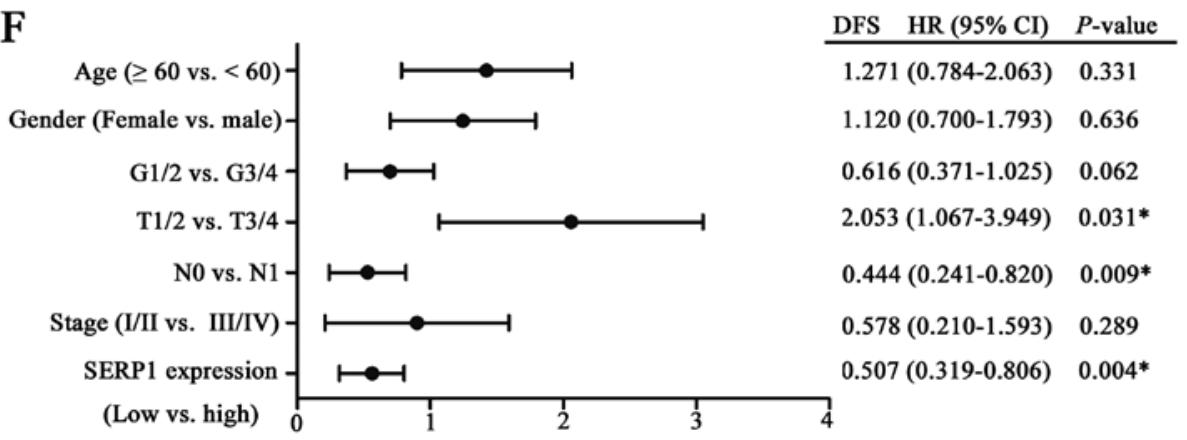

Figure 4. The correlation between expression level of the stress associated endoplasmic reticulum protein 1 (SERP1) and prognosis of pancreatic ductal adenocarcinoma (PDAC) patients. (A) K-M curve for OS of patients with high ( $\mathrm{n}=89)$ and low $(\mathrm{n}=87)$ SERP1 expression level. Results revealed that high SERP1 expression level was correlated with shorter OS for PDAC patients. (B) SERP1 high expression group ( $\mathrm{n}=61)$ had shorter DFS than SERP1 low expression group $(\mathrm{n}=76)$. (C) The expression of SERP1 in OS-good and poor patients. (D) The expression of SERP1 in DFS-good and poor patients. (E and F) Multivariate Cox regression analyses showed SERP1 expression was an independent factor of patients' overall survival and disease-free survival.

results showed that downregulated SERP1 promoted cell apoptosis probably via upregulating SRPRB expression.

$S R P R B$ promotes cell apoptosis through $N F-\kappa B$ activation. $\mathrm{NF}-\kappa \mathrm{B}$ activation plays an important role in controlling the survival of tumor cells (20-22), the abnormal upregulation were thought to promote tumor cell survival (23). In the present study, we found that gene sets related to NF- $\kappa B$ signaling pathway were enriched in PDAC tissues by GSEA (Fig. 8A). Moreover, the expression level of NF- $\mathrm{KB}$ was positively correlated with the SERP1 expression $(\mathrm{P}<0.0001)$ (Fig. 8B) and negatively correlated with SRPRB in 178 PDAC patients from TCGA database $(\mathrm{P}=0.0090)$ (Fig. 8C). To validate that upregulated SRPRB can suppress the expression of NF- $\mathrm{KB}$, pcDNA3.0-SRPRB was transfected into PANC-1 cells and

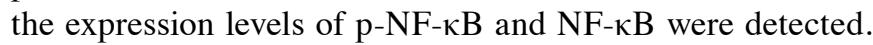




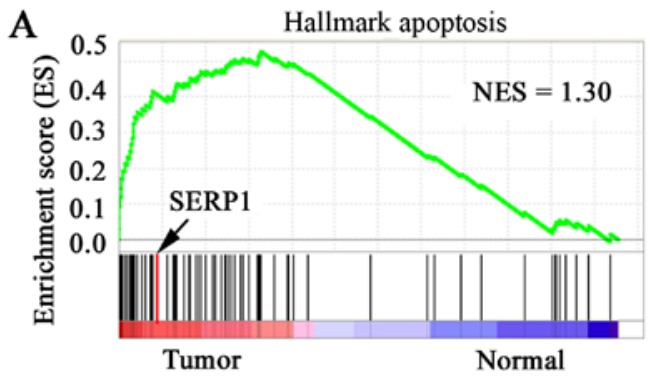

C

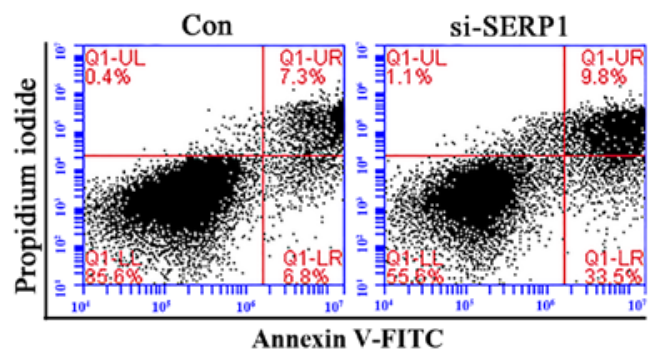

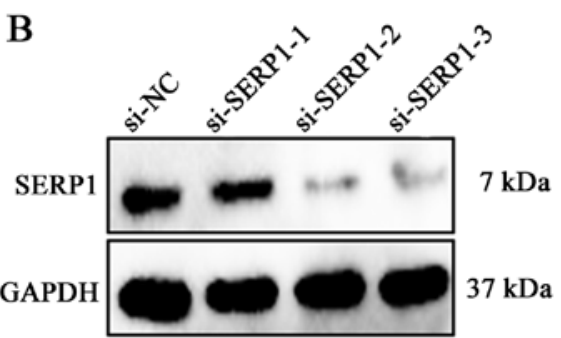

D

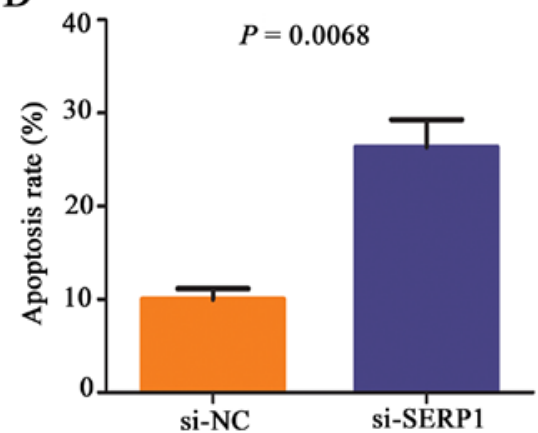

Figure 5. Stress associated endoplasmic reticulum protein 1 (SERP1) is mainly involved in regulating cell apoptosis. (A) Apoptosis gene sets were enriched in tumor group. The curve is the enrichment score of these genes by GSEA. (B) PANC-1 cells were transfected with three SERP1 siRNA. Forty-eight hours after transfection, the expression level of SERP1 was detected by western blotting, and results revealed that the expression level of SERP1 was obviously downregulated in PANC-1 cells transfected with SERP1-siRNA compared to negative control cells. (C and D) Compared to control group, PANC-1 cells transfected with SERP1-siRNA had a higher rate of apoptosis.

A

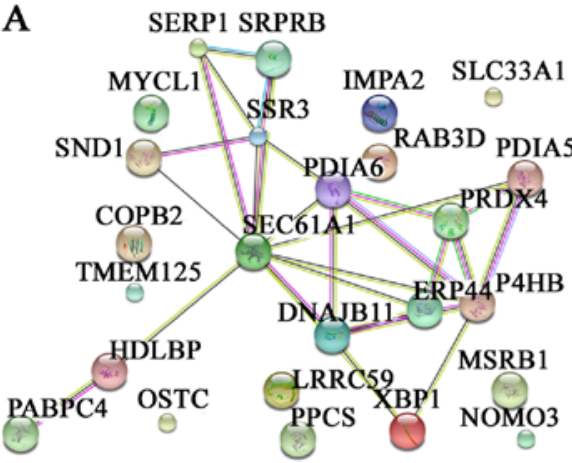

C

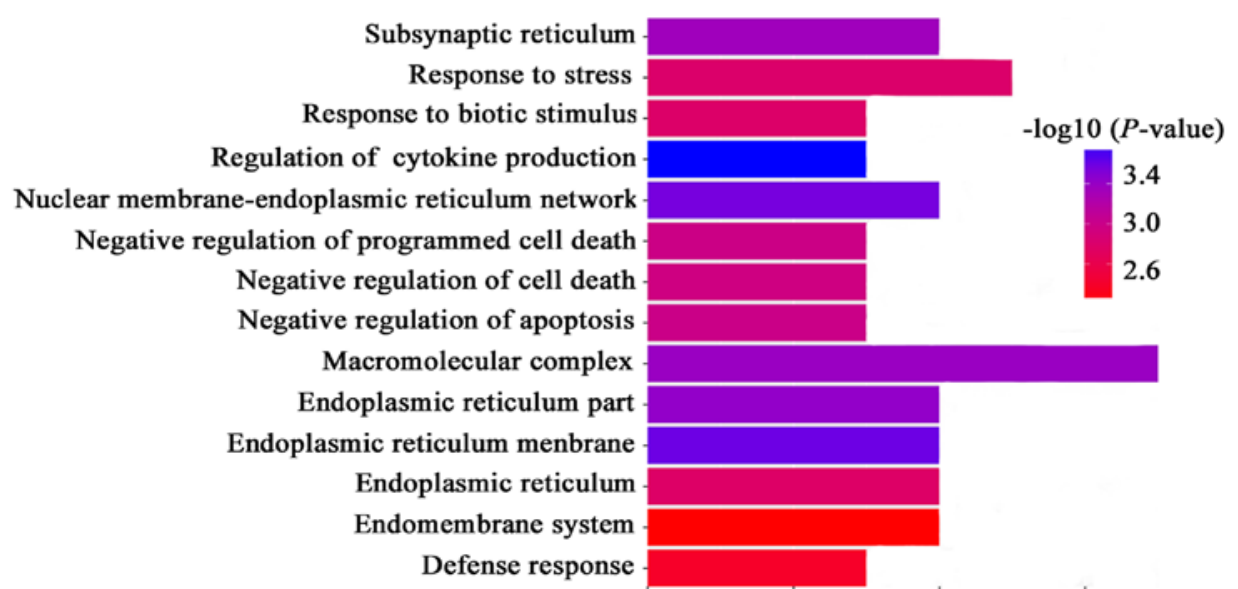

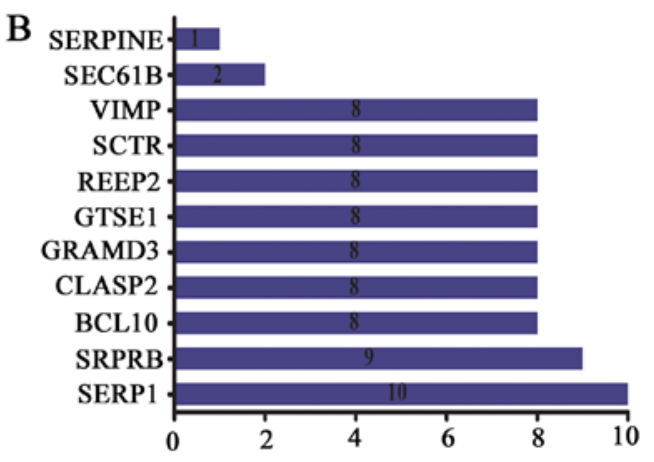

Figure 6. Protein-protein interaction (PPI) and GO analyses for interaction genes with the stress associated endoplasmic reticulum protein 1 (SERP1). (A and B) PPI analysis of interaction genes with SERP1. SRP receptor $\beta$ subunit (SRPRB) was the main node protein for the interaction network. (C) GO analysis of the interaction gene with SERP1, and these genes were mainly involved in regulating cell death and apoptosis. 

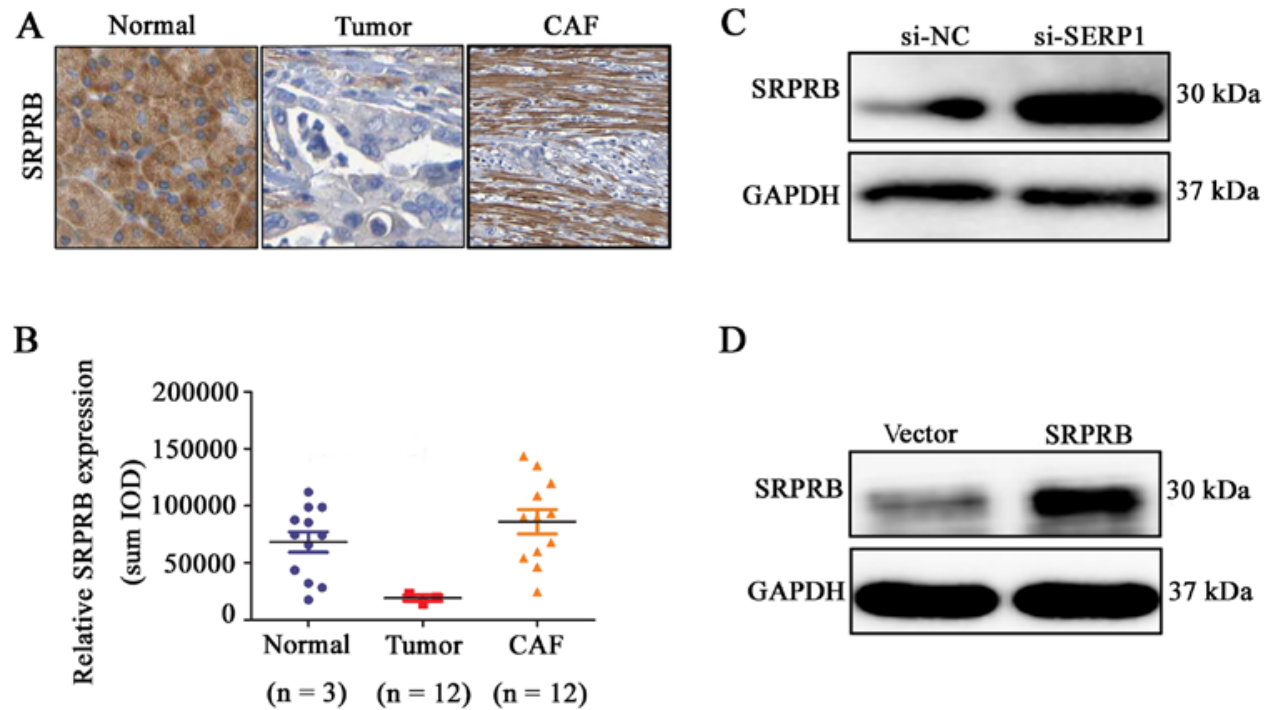

D

$\mathrm{E}$
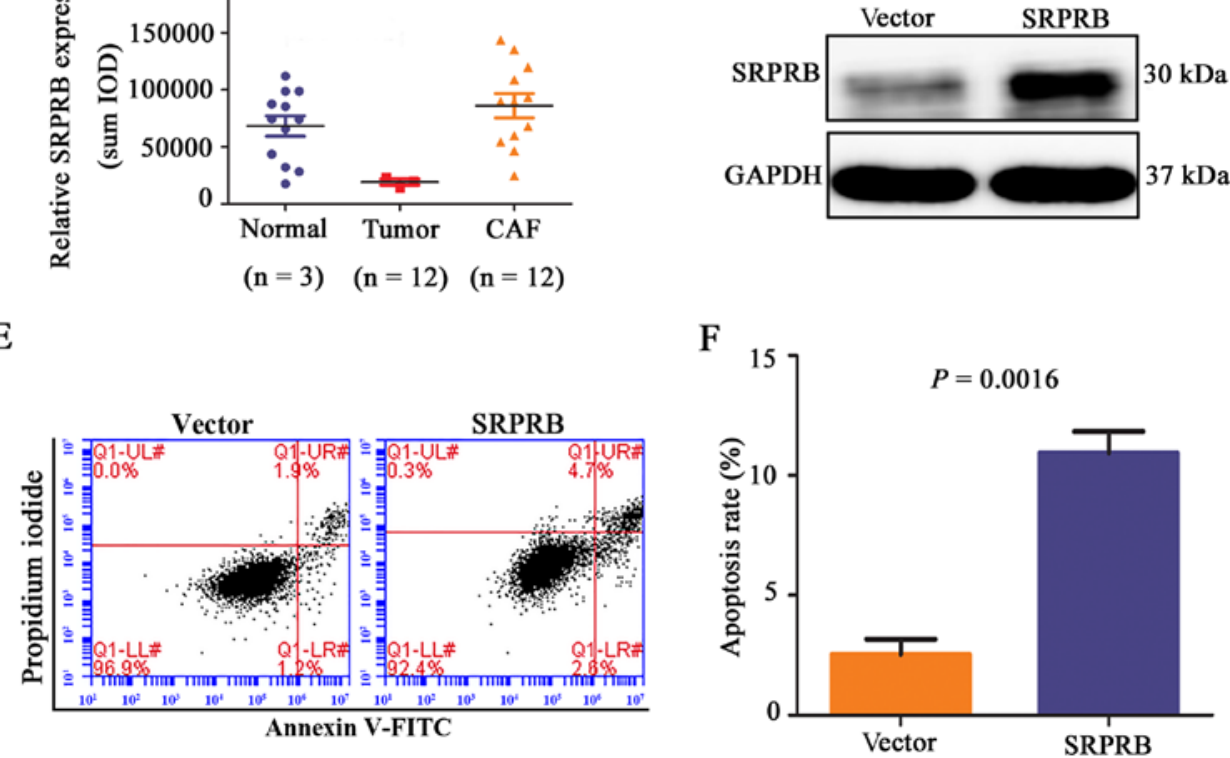

Figure 7. Downregulated stress associated endoplasmic reticulum protein 1 (SERP1) promotes apoptosis via increasing SRP receptor $\beta$ subunit (SRPRB) expression. (A and B) The Human Protein Atlas database results revealed that the expression level of SRPRB were upregulated in stromal fibroblast compared with tumor tissues. (C) Western blot analysis confirmed that downregulated SERP1 can increase the expression of SRPRB in PANC-1 cells. (D) PANC-1 cells were transfected with SRPRB overexpression plasmid, and western blot analysis found that the expression level of SRPRB was significantly increased in PANC-1 cells. (E and F) Compared to control group, SRPRB overexpression significantly promoted apoptosis of PANC-1 cells.
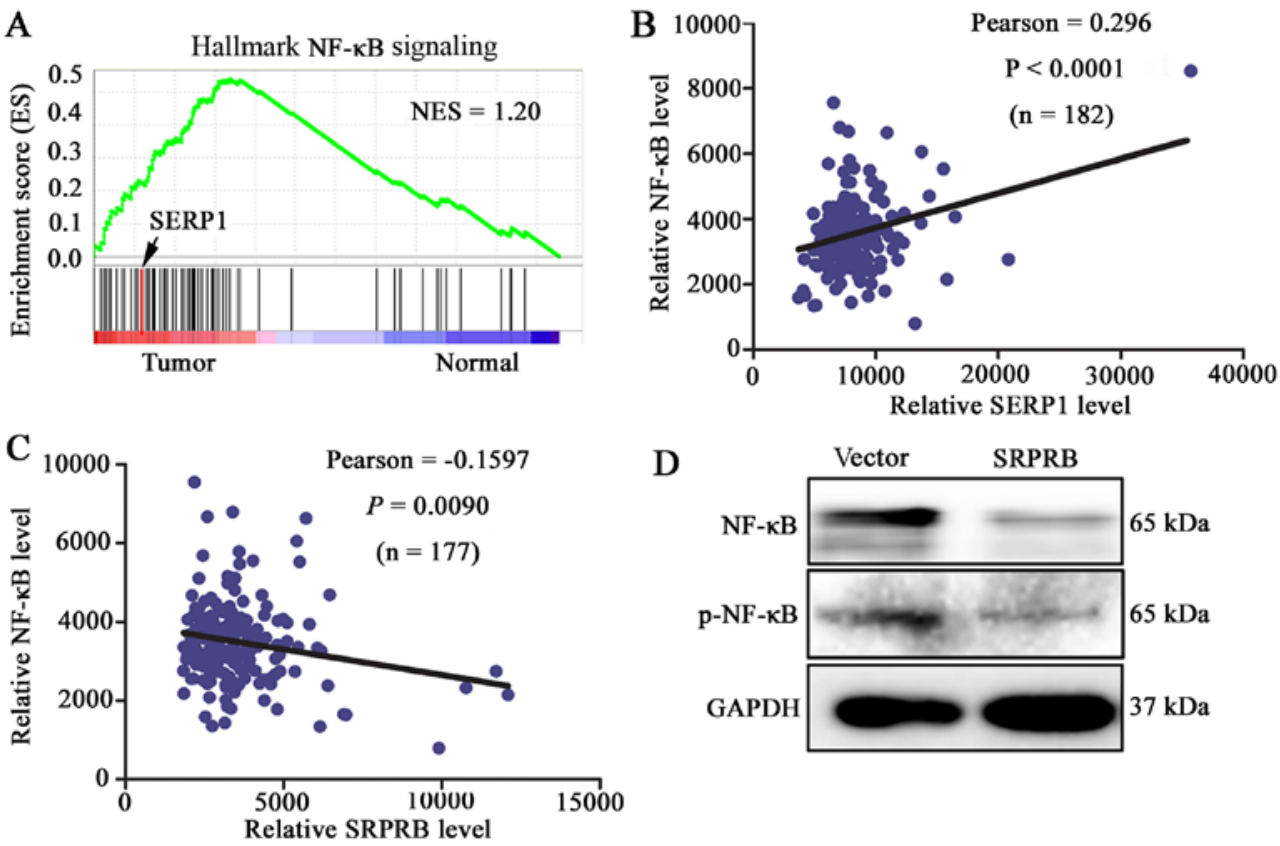

D

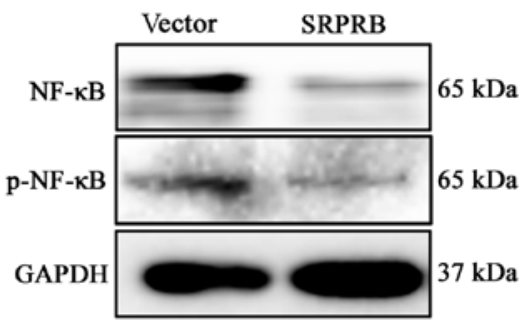

Figure 8. Downregulated stress associated endoplasmic reticulum protein 1 (SERP1) promotes cell apoptosis via regulating SRP receptor $\beta$ subunit (SRPRB)

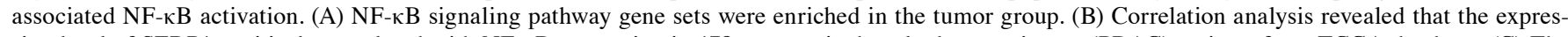
sion level of SERP1 positively correlated with NF-kB expression in 178 pancreatic ductal adenocarcinoma (PDAC) patients from TCGA database. (C) The expression level of SRPRB was negatively correlated with NF- $\mathrm{kB}$ expression in 178 PDAC patients from TCGA database. (D) Western blotting confirmed that

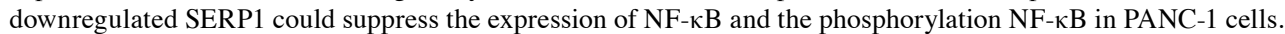


Table I. Co-expression genes with SERP1 from cBioPortal database.

\begin{tabular}{lccc}
\hline $\begin{array}{l}\text { Gene } \\
\text { symbol }\end{array}$ & Cytoband & $\begin{array}{c}\text { Pearson } \\
\text { score }\end{array}$ & $\begin{array}{c}\text { Spearman } \\
\text { score }\end{array}$ \\
\hline SSR3 & 3q25.31 & 0.83 & 0.61 \\
XBP1 & 22q12.1122q12 & 0.71 & 0.31 \\
SEC61A1 & 3q21.3 & 0.71 & 0.46 \\
HDLBP & $2 \mathrm{q} 37.3$ & 0.7 & 0.49 \\
IMPA2 & $18 \mathrm{p} 11.2$ & 0.68 & 0.36 \\
SLC33A1 & $3 \mathrm{q} 25.31$ & 0.68 & 0.44 \\
PABPC4 & $1 \mathrm{p} 34.2$ & 0.67 & 0.34 \\
RAB3D & $19 \mathrm{p} 13.2$ & 0.67 & 0.29 \\
MYCL & $1 \mathrm{p} 34.2$ & 0.66 & 0.21 \\
OSTC & $4 \mathrm{q} 25$ & 0.66 & 0.52 \\
P4HB & $17 \mathrm{q} 25$ & 0.65 & 0.36 \\
TMEM125 & $1 \mathrm{p} 34.2$ & 0.65 & 0.22 \\
COPB2 & $3 \mathrm{q} 23$ & 0.63 & 0.5 \\
MSRB1 & $16 \mathrm{p} 13.3$ & 0.63 & 0.43 \\
LRRC59 & $17 \mathrm{q} 21.33$ & 0.63 & 0.43 \\
SRPRB & $3 \mathrm{q} 22.1$ & 0.63 & 0.55 \\
PPCS & $1 \mathrm{p} 34.2$ & 0.62 & 0.33 \\
ERP44 & $9 \mathrm{q} 31.1$ & 0.62 & 0.37 \\
SND1 & $7 \mathrm{q} 31.3$ & 0.62 & 0.37 \\
PRDX4 & Xp22.11 & 0.61 & 0.39 \\
PDIA5 & 3q21.1 & 0.61 & 0.35 \\
PDIA6 & 2p25.1 & 0.6 & 0.44 \\
NOMO3 & $16 \mathrm{p} 13$ & 0.6 & 0.28 \\
DNAJB11 & $3 \mathrm{q} 27.3$ & 0.6 & 0.55 \\
\hline
\end{tabular}

The results demonstrated that the expression levels of the phosphorylation NF- $\kappa \mathrm{B}$ and $\mathrm{NF}-\kappa \mathrm{B}$ were reduced with SRPRB upregulation in PANC-1 cells (Fig. 8D). The above data suggested that downregulated SERP1 prompted apoptosis possibly via upregulating SRPRB expression and inhibiting the expression of p-NF- $\mathrm{kB}$ and NF- $\mathrm{KB}$ in PDAC cells.

\section{Discussion}

In the present study, the prognostic value of SERP1 in PDAC is reported. We demonstrated that SERP1 expression level was significantly upregulated in human PDAC tissues compared to adjacent noncancerous tissues. High expression of SERP1 was positively associated with advanced clinical stage and shorter survival time (OS and DFS), indicating poor prognosis in PDAC. In vitro, downregulated SERP1 expression significantly promoted PANC-1 cell apoptosis which was mainly carried out by upregulating SRPRB expression and inhibiting the activation of NF- $\kappa$ B. Therefore, we speculated SERP1 may be a novel prognosis marker of PDAC patients via anti-apoptosis, which may be associated with the expression of SRPRB.

A growing number of new biomarkers have been found, such as noncoding RNA, microRNAs and liquid biopsies. Among these biomarkers, $\mathrm{C}$-reactive protein, mutational status of P53, KRAS or BRCA2 are the most useful biomarkers in clinical practice (24). However, prognostic markers for pancreatic cancer are still limited in clinical practice. Previous studies confirmed a significant correlation between high SERP1 levels and poor patient outcome in glioblastoma patients (25), no studies were found in other tumors. In present study, we first found that SERP1 was strongly correlated with prognosis of PDAC patients. A previous study found that SERP1 expression level was enhanced in vitro by hypoxia and/or reoxygenation or other forms of stress, and associated with unfolded proteins in endoplasmic reticulum (ER) stress (26). Many different physiological processes, highly secretory cells such as pancreatic $\beta$-cells, plasma B lymphocytes, and pathological conditions such as hypoxia, ER $\mathrm{Ca}^{2+}$ depletion, and cancer can cause an imbalance between ER protein folding load and capacity, leading to accumulation of unfolded proteins in the ER lumen, a condition known as 'ER stress' (27). In addition, SERP1 reintroduction could reverse the hypoxic cell death and plays an important role in promoting tumor cell survival (25). The status of ER stress control early biogenesis of membrane proteins (28), which is very important in cell death, for example, inhibition of ER stress leads to rescue of triptolide-mediated pancreatic cancer MIAPaCa-2 cell death (27). In this study, PPI and GO analysis suggested that SERP1 and its interactional proteins are mainly involved in regulating cell death and apoptosis, and we observed that downregulated SERP1 expression actually promoted cell apoptosis compared with negative control group in vitro, which may be a potential cause of influencing prognosis of PDAC patients.

We found that SRPRB was an important interactional protein in the whole PPI network, and hypothesized that SRPRB may have an important role in regulating cell apoptosis. SRPRB is a novel human gene whose transcripts were upregulated in apoptotic MCF-7 cells (29), and generally localized in the cytoplasm. Tissue-specific expression of SRPRB was found in various human tumors. For example, positive staining of SRPRB was found in the liver, lung, breast, colon, stomach, esophagus and testis, exhibited a ubiquitous expression pattern while its expression was upregulated in tumor tissues compared with corresponding normal tissues (30). As another study reported, both the normal and tumor tissues of ovary were absent of SRPRB expression (30). By contrast, in the present study, we found that the expression level of SRPRB was significantly downregulated in PDAC tissues, and significantly upregulated in stromal fibroblasts. Previous studies found SRPRB plays certain biological role in the regulation of cell proliferation and apoptosis, SRPRB induced inhibition of HHCC growth and cell cycle through regulating genes such as p21 and TIMP3 (31). In vitro, we actually observed that SRPRB overexpression obviously increased cell apoptosis rate, and its expression level could be regulated by SERP1. In addition, correlation analysis found that NF- $\mathrm{kB}$ were significantly upregulated in PDAC tissues, and positively correlated with SERP1 level and negatively correlated with SRPRB level. NF- $\kappa B$ activation plays a critical role in regulating cell survival (32), it has been proved that downregulation of $\mathrm{NF}-\kappa \mathrm{B}$ mediated cell apoptosis (33). For example, inhibition of prosurvival Akt/NF- $\kappa B$ signaling induced apoptosis in prostate cancer cells (34), ovarian cancer cells (35) and astroglioma cells (36). Activation of NF- $\mathrm{kB}$ mainly occurs via 
phosphorylation of $\mathrm{NF}-\kappa \mathrm{B}$ proteins, such as $\mathrm{p} 65$, within their transactivation domain by a variety of kinases in response to distinct stimuli, and then enhanced cell anti-apoptosis ability (37). In fact, phosphorylated p65 was significantly upregulated in PDAC cell lines, and associated with cell proliferation, cell cycle, and apoptosis (38). Moreover, specific targeted inhibition the phosphorylation $N F-\kappa B$ pathways could induce cell apoptosis (39). In this study, we found that SRPRB overexpression obviously decreased the expression of $\mathrm{NF}-\kappa \mathrm{B}$ and the phosphorylation NF- $\mathrm{B}$. Hence, we assumed that downregulated SERP1 may promote PDAC cell apoptosis via inhibiting NF- $\mathrm{BB}$ activation, which may be associated with SRPRB expression. We considered that anti-apoptosis induced by SERP1 may be one of the important mechanisms that could promote tumor progression, and affect the prognosis of patients, which has not been previously reported. Although we have demonstrated the relationship between SERP1 and prognosis as well as its possible mechanism, great number of clinical sample data are still need.

In conclusion, SERP1 is a novel potential prognostic marker for PDAC, and downregulated SERP1 expression promoted PDAC cell apoptosis and inhibited NF- $\mathrm{B}$ activation probably by upregulating SRPRB expression. The present study provided a novel pathogenetic mechanism and a new treatment direction for PDAC. To the best of our knowledge, this is the first study showing the prognostic value of SERP1 for PDAC patients.

\section{Acknowledgements}

This study was supported by the Special Foundation for Scientific Research in the Public Interest by the National Health and Family Planning Commission of China (no. 201402001), CAMS Innovation Fund for Medical Sciences (no. 2016-I2M-1-002), the National Natural Science Foundation of China (no. 31471366).

\section{References}

1. Martinez-Useros J and Garcia-Foncillas J: The role of BRCA2 mutation status as diagnostic, predictive, and prognosis biomarker for pancreatic cancer. Biomed Res Int 2016: 1869304, 2016.

2. Swords DS, Firpo MA, Scaife CL and Mulvihill SJ: Biomarkers in pancreatic adenocarcinoma: Current perspectives. Onco Targets Ther 9: 7459-7467, 2016.

3. Pietrasz D, Pécuchet N, Garlan F, Didelot A, Dubreuil O, Doat S, Imbert-Bismut F, Karoui M, Vaillant JC, Taly V, et al: Plasma circulating tumor DNA in pancreatic cancer patients is a prognostic marker. Clin Cancer Res 23: 116-123, 2017.

4. Tanouchi A, Taniuchi K, Furihata M, Naganuma S, Dabanaka K, Kimura M, Watanabe R, Kohsaki T, Shimizu T, Saito M, et al: CCDC88A, a prognostic factor for human pancreatic cancers, promotes the motility and invasiveness of pancreatic cancer cells. J Exp Clin Cancer Res 35: 190, 2016.

5. Sun B, Liu X, Gao Y, Li L and Dong Z: Downregulation of miR-124 predicts poor prognosis in pancreatic ductal adenocarcinoma patients. Br J Biomed Sci 73: 152-157, 2016.

6. Nuzhat Z, Kinhal V, Sharma S, Rice GE, Joshi V and Salomon C: Tumour-derived exosomes as a signature of pancreatic cancer liquid biopsies as indicators of tumour progression. Oncotarget 8 : 17279-17291, 2017.

7. Chandra Gupta S and Nandan Tripathi Y: Potential of long noncoding RNAs in cancer patients: From biomarkers to therapeutic targets. Int J Cancer 140: 1955-1967, 2017.

8. Yang D, Shi J, Fu H, Wei Z, Xu J, Hu Z, Zhang Y, Yan R and Cai Q: Integrin $\beta 1$ modulates tumour resistance to gemcitabine and serves as an independent prognostic factor in pancreatic adenocarcinomas. Tumour Biol 37: 12315-12327, 2016.
9. Li Q, Wang H, Zogopoulos G, Shao Q, Dong K, Lv F, Nwilati K, Gui XY, Cuggia A, Liu JL, et al: Reg proteins promote acinarto-ductal metaplasia and act as novel diagnostic and prognostic markers in pancreatic ductal adenocarcinoma. Oncotarget 7: 77838-77853, 2016.

10. Tsuboi M, Taniuchi K, Furihata M, Naganuma S, Kimura M, Watanabe R, Shimizu T, Saito M, Dabanaka K, Hanazaki K, et al: Vav3 is linked to poor prognosis of pancreatic cancers and promotes the motility and invasiveness of pancreatic cancer cells. Pancreatology 16: 905-916, 2016.

11. Li X, Xiao Y, Fan S, Xiao M, Wang X, Zhu X, Chen X, Li C, Zong G,Zhou G, et al: Overexpression of DIXDC1 correlates with enhanced cell growth and poor prognosis in human pancreatic ductal adenocarcinoma. Hum Pathol 57: 182-192, 2016.

12. Hori O, Miyazaki M, Tamatani T, Ozawa K, Takano K, Okabe M, Ikawa M, Hartmann E, Mai P, Stern DM, et al: Deletion of SERP1/RAMP4, a component of the endoplasmic reticulum (ER) translocation sites, leads to ER stress. Mol Cell Biol 26: 4257-4267, 2006.

13. Kim HS, Chang I, Kim JY, Choi KH and Lee MS: Caspasemediated p65 cleavage promotes TRAIL-induced apoptosis. Cancer Res 65: 6111-6119, 2005.

14. Guzmán EA, Maers K, Roberts J, Kemami-Wangun HV, Harmody D and Wright AE: The marine natural product microsclerodermin A is a novel inhibitor of the nuclear factor kappa B and induces apoptosis in pancreatic cancer cells. Invest New Drugs 33: 86-94, 2015.

15. Mak P, Li J, Samanta $S$ and Mercurio AM: ER $\beta$ regulation of $\mathrm{NF}-\kappa \mathrm{B}$ activation in prostate cancer is mediated by HIF-1. Oncotarget 6: 40247-40254, 2015.

16. De Simone V, Franzè E, Ronchetti G, Colantoni A, Fantini MC, Di Fusco D, Sica GS, Sileri P, MacDonald TT, Pallone F, et al: Th17-type cytokines, IL-6 and TNF- $\alpha$ synergistically activate STAT3 and NF- $\kappa$ B to promote colorectal cancer cell growth. Oncogene 34: 3493-3503, 2015.

17. Dolcet X, Llobet D, Pallares J and Matias-Guiu X: NF- $\kappa B$ in development and progression of human cancer. Virchows Arch 446: 475-482, 2005.

18. Ma Q, Peng Z, Wang L, Li Y, Wang K, Zheng J, Liang Z and Liu T: miR-19a correlates with poor prognosis of clear cell renal cell carcinoma patients via promoting cell proliferation and suppressing PTEN/SMAD4 expression. Int J Oncol 49: 2589-2599, 2016.

19. Kong FY, Wei X, Zhou K, Hu W, Kou YB, You HJ, Liu XM, Zheng KY and Tang RX: Bioinformatics analysis reveals distinct molecular characteristics of hepatitis B-related hepatocellular carcinomas from very early to advanced Barcelona clinic liver cancer stages. PLoS One 11: e0158286, 2016.

20. Chen Y, Yang Y, Sun M, Yan Z, Wu L, Cui X, Zhang G, Morris SW and Zhang Q: Inhibition of caspase- 8 activity caused by overexpression of BCL10 contributes to the pathogenesis of high-grade MALT lymphoma. Pediatr Blood Cancer 58: 865-871, 2012.

21. Staudt LM: Oncogenic activation of NF-kappaB. Cold Spring Harb Perspect Biol 2: a000109, 2010.

22. Kuo SH, Chou CH, Cheng AL, Wang CW, Chen $\mathrm{YH}$ and Chen RJ: Expression of BCL10 in cervical cancer has a role in the regulation of cell growth through the activation of NF- $\kappa \mathrm{B}$-dependent cyclin D1 signaling. Gynecol Oncol 126: 245-251, 2012.

23. Li Z, Zhang H, Chen Y, Fan L and Fang J: Forkhead transcription factor FOXO3a protein activates nuclear factor $\kappa \mathrm{B}$ through B-cell lymphoma/leukemia 10 (BCL10) protein and promotes tumor cell survival in serum deprivation. J Biol Chem 287: 17737-17745, 2012.

24. Martinez-Useros J and Garcia-Foncillas J: Can molecular biomarkers change the paradigm of pancreatic cancer prognosis? BioMed Res Int 2016: 4873089, 2016.

25. Mucaj V, Lee SS, Skuli N, Giannoukos DN, Qiu B, EisingerMathason TS, Nakazawa MS, Shay JE, Gopal PP, Venneti S, et al: MicroRNA-124 expression counteracts pro-survival stress responses in glioblastoma. Oncogene 34: 2204-2214, 2015.

26. Yamaguchi A, Hori O, Stern DM, Hartmann E, Ogawa S and Tohyama M: Stress-associated endoplasmic reticulum protein 1 (SERP1)/Ribosome-associated membrane protein 4 (RAMP4) stabilizes membrane proteins during stress and facilitates subsequent glycosylation. J Cell Biol 147: 1195-1204, 1999.

27. Mujumdar N, Banerjee S, Chen Z, Sangwan V, Chugh R, Dudeja V, Yamamoto M, Vickers SM and Saluja AK: Triptolide activates unfolded protein response leading to chronic ER stress in pancreatic cancer cells. Am J Physiol Gastrointest Liver Physiol 306: G1011-G1020, 2014. 
28. Faria D, Lentze N, Almaça J, Luz S, Alessio L, Tian Y, Martins JP, Cruz P, Schreiber R, Rezwan M, et al: Regulation of $\mathrm{ENaC}$ biogenesis by the stress response protein SERP1. Pflugers Arch 463: 819-827, 2012.

29. Yan W, Wang WL, Zhu F, Chen SQ, Li QL and Wang L: Isolation of a novel member of small $\mathrm{G}$ protein superfamily and its expression in colon cancer. World J Gastroenterol 9: 1719-1724, 2003.

30. Zhang Y, Li Q, Zhu F, Cui J, Li K, Li Q, Wang R, Wang W, Wang W and Yan W: Subcellular localization of APMCF1 and its biological significance of expression pattern in normal and malignant human tissues. J Exp Clin Cancer Res 28: 111, 2009.

31. Li Q, Yan W, Cheng S, Guo S, Wang W, Zhang Z, Wang L, Zhang $\mathrm{J}$ and Wang $\mathrm{W}$ : Introduction of $\mathrm{G} 1$ phase arrest in human hepatocellular carcinoma cells (HHCC) by APMCF1 gene transfection through the downregulation of TIMP3 and upregulation of the CDK inhibitors p21. Mol Biol Rep 33: 257-263, 2006.

32. Yu M, Chen Y, He Y, Podd A, Fu G, Wright JA, Kleiman E, Khan WN, Wen R and Wang D: Critical role of B cell lymphoma 10 in BAFF-regulated NF- $\kappa \mathrm{B}$ activation and survival of anergic B cells. J Immunol 189: 5185-5193, 2012.

33. Kadirareddy RH, Vemuri SG and Palempalli UM: Probiotic conjugated linoleic acid mediated apoptosis in breast cancer cells by downregulation of NFאB. Asian Pac J Cancer Prev 17: 3395-3403, 2016.
34. Liu Y, Gao X, Deeb D, Zhang Y, Shaw J, Valeriote FA and Gautam SC: Mycotoxin verrucarin A inhibits proliferation and induces apoptosis in prostate cancer cells by inhibiting prosurvival Akt/NF-kB/mTOR signaling. J Exp Ther Oncol 11: 251-260, 2016

35. Yang J, Li G and Zhang K: Pro-survival effects by NF- $\kappa \mathrm{B}$, Akt and ERK(1/2) and anti-apoptosis actions by Six1 disrupt apoptotic functions of TRAIL-Dr4/5 pathway in ovarian cancer. Biomed Pharmacother 84: 1078-1087, 2016.

36. Xia W, Tian H, Cai X, Kong H, Fu W, Xing W, Wang Y, Zou M, $\mathrm{Hu} \mathrm{Y}$ and $\mathrm{Xu}$ D: Inhibition of SUMO-specific protease 1 induces apoptosis of astroglioma cells by regulating NF- $\kappa \mathrm{B} / \mathrm{Akt}$ pathways. Gene 595: 175-179, 2016.

37. Zhang L, Shao L, Creighton CJ, Zhang Y, Xin L, Ittmann M and Wang J: Function of phosphorylation of NF- $\kappa \mathrm{B}$ p65 ser536 in prostate cancer oncogenesis. Oncotarget 6: 6281-6294, 2015.

38. Hu YQ, Si LJ, Ye ZS, Lin ZH and Zhou JP: Inhibitory effect of ARHI on pancreatic cancer cells and NF- $\kappa$ B activity. Mol Med Rep 7: 1180-1184, 2013.

39. Li W, Wu J, Li Z, Zhou Z, Zheng C, Lin L, Tan B, Huang M and Fan M: Melatonin induces cell apoptosis in Mia PaCa-2 cells via the suppression of nuclear factor- $\mathrm{K} B$ and activation of ERK and JNK: A novel therapeutic implication for pancreatic cancer. Oncol Rep 36: 2861-2867, 2016. 